\title{
Hydrogen storage capacity of Ti-doped boron-nitride and B/Be-substituted carbon nanotubes
}

\author{
E. Durgun, ${ }^{1,2}$ Y.-R. Jang, ${ }^{1,3}$ and S. Ciraci ${ }^{1,2, *}$ \\ ${ }^{1}$ Department of Physics, Bilkent University, Ankara 06800, Turkey \\ ${ }^{2}$ National Nanotechnology Research Center, Bilkent University, Ankara 06800, Turkey \\ ${ }^{3}$ Department of Physics, University of Incheon, Incheon 402-749, Korea
}

(Received 29 March 2007; published 27 August 2007)

\begin{abstract}
We investigate the hydrogen absorption capacity of two tubular structures, namely, B/Be-substituted singlewall carbon nanotube (SWNT) and Ti covered single-wall boron nitride nanotube (SWBNT) using firstprinciples plane wave method. The interaction of $\mathrm{H}_{2}$ molecules with the outer surface of bare SWBNT, which is normally very weak, can be significantly enhanced upon functionalization by $\mathrm{Ti}$ atoms. Each $\mathrm{Ti}$ atom adsorbed on SWBNT can bind up to four $\mathrm{H}_{2}$ molecules with an average binding energy suitable for room temperature storage. While the substitution process of Be atom on SWNT is endothermic, the substituted Be strengthens the interaction between tube surface and $\mathrm{H}_{2}$ to hold one $\mathrm{H}_{2}$ molecule.
\end{abstract}

DOI: 10.1103/PhysRevB.76.073413

PACS number(s): 73.22.-f, 73.63.Nm, 75.75.+a

\section{INTRODUCTION}

Safe and efficient hydrogen storage requires gravimetric density exceeding $6 \mathrm{wt} \%$ together with fast and reversible kinetics of absorption and desorption. ${ }^{1,2}$ Nanostructures or porous substances having high surface to volume ratios have been favored for the high capacity storage medium.

There are several studies focused on carbon-based materials such as nanotubes, ${ }^{3-8}$ fullerenes (i.e., $\left.\mathrm{C}_{60}\right),{ }^{9,10}$ metal hydrides and metal-organic frameworks, ${ }^{11,12}$ titanium metallocarbohedryne, ${ }^{13,14}$ polyacethylene, ${ }^{15}$ and transitionmetal-ethylene complexes. ${ }^{16}$ While the binding of hydrogen molecule on the outer surface of the bare single-wall carbon nanotube (SWNT) is very weak, ${ }^{7}$ a single Ti atom adsorbed on $(8,0)$ SWNT can bind up to four $\mathrm{H}_{2} \cdot{ }^{8}$ It is found that a $(8,0)$ SWNT can store hydrogen molecules up to $8 \mathrm{wt} \%$, exceeding the minimum requirement set for practical applications. Similar storage capacity has been obtained for $\mathrm{Ti}$ atom adsorbed on $\mathrm{C}_{60} \cdot{ }^{9,10}$ The binding of $\mathrm{H}_{2}$ molecules has been explained by a simple Dewar-Chatt-Duncanson model. ${ }^{17}$

Recent analysis for carbon fullerenes revealed that the binding energy of hydrogen molecules can be enhanced significantly $(0.2-0.4 \mathrm{eV})$ by substitution with $\mathrm{B}$ or Be atoms. ${ }^{18}$ Furthermore, experiments showed that boron nitride nanotubes can absorb 1.8-2.6 wt \% hydrogen at about $10 \mathrm{MPa}$ at room temperature. ${ }^{19,20}$ Moreover, the possibility of covering the exterior surface of single-wall boron nitride nanotubes (SWBNTs) up to $50 \%$ by hydrogen was presented. ${ }^{21} \mathrm{~A}$ chemisorption calculation of hydrogen molecules in $\mathrm{BN}$ clusters showed that they would be one of the possible hydrogen storage media. ${ }^{22}$

Motivated with these results, we examined hydrogen storage capacity of bare and Ti adsorbed SWBNT. We also investigated the $\mathrm{H}_{2}$ storage capacity of B- and Be-substituted SWNTs. This Brief Report will be a supplement to our results published earlier ${ }^{7,8,10,16}$ and will further clarify the interaction between $\mathrm{H}_{2}$ and nanotubes.

We have performed first-principles plane wave calculations $^{23}$ within density functional theory ${ }^{24}$ (DFT) using ultrasoft pseudopotentials. ${ }^{25}$ The exchange correlation poten- tial has been approximated by generalized gradient approximation (GGA) ${ }^{26}$ All structures have been treated within supercell geometry using the periodic boundary conditions with dimensions $20 \times 20 \times c_{o}$ [ $c_{o}$ is the relaxed lattice constant of nanotubes along the tube axis and is equal to 4.26 and $4.33 \AA$ for $(8,0)$ SWNT and $(8,0)$ SWBNT, respectively]. In self-consistent total energy calculations the Brillouin zone is sampled in the $\mathbf{k}$ space within the Monkhorst-Pack scheme ${ }^{27}$ by $1 \times 1 \times 15$ mesh points. A plane basis set with kinetic energy of $400 \mathrm{eV}$ has been used. All atomic positions and lattice parameters are optimized by using conjugate gradient method where total energy and atomic forces are minimized. The convergence for energy is chosen as $10^{-5} \mathrm{eV}$ between two ionic steps, and the maximum force allowed on each atoms is $0.05 \mathrm{eV} / \AA$.

\section{ABSORPTION OF $\mathrm{H}_{2}$ MOLECULE ON BARE AND Ti COVERED $(8,0)$ SINGLE-WALL BORON NITRIDE NANOTUBE}

SWBNTs have attracted much interest since they possess unique electronic and mechanical properties. For a given chiral angle and approximately equal radii (or index $n$ ), SWBNT and $\mathrm{SWNT}^{28}$ exhibit similar structure, such as zigzag $(n, 0)$ or armchair $(n, n)$. SWBNT can be viewed as SWNT, in which the alternating $\mathrm{C}$ atoms are replaced by $\mathrm{B}$ and $\mathrm{N}$ atoms. However, owing to the charge transfer between $\mathrm{B}$ and $\mathrm{N}$ (and hence induced ionic character of the $\mathrm{B}-\mathrm{N}$ bonds), SWBNT exhibit physical properties which are dramatically different than those of SWNT. For example, SWBNTs have higher heat tolerance and are less likely to oxidize. $^{29}$ Moreover, SWBNTs are always semiconductor with uniform and wide band gap no matter what their radii and chirality are. ${ }^{30}$ The experimentally synthesized SWBNTs were observed to have a diameter ranging from 0.5 to $1.2 \mathrm{~nm} .{ }^{29,31}$ It was also reported that synthesis of the zigzag-type SWBNT is more favorable to that of the armchair type. ${ }^{32,33}$ Recent studies have indicated that SWBNTs can be a highly efficient medium for hydrogen storage. ${ }^{19-22}$ It is suggested that the binding energy of hydrogen molecule on SWBNT may increase as much as $40 \%$ compared with 

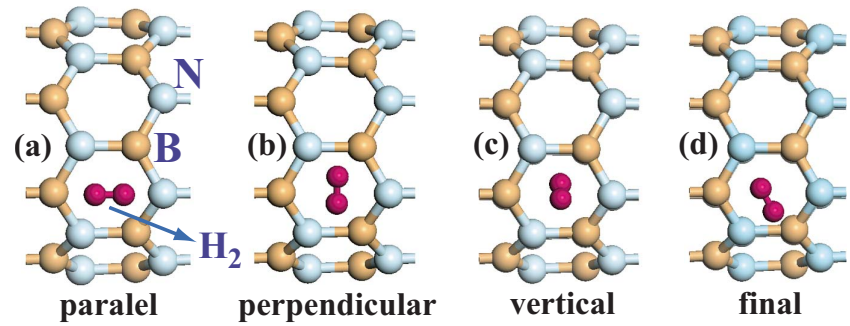

FIG. 1. (Color online) Possible $\mathrm{H}_{2}$ absorption geometries on $(8,0)$ SWBNT. [(a)-(c)] Various initial configurations of $\mathrm{H}_{2}$ absorbing at hollow site. (d) Final configuration of $\mathrm{H}_{2}$ after structure optimization.

that on SWNT due to the heteropolar bonding. ${ }^{34}$

We first investigate the interaction between $\mathrm{H}_{2}$ and the outer surface of $(8,0) \mathrm{SWBNT}$, which is chosen as prototype. To this end, we consider $\mathrm{H}_{2}$ absorbed at different sites (hollow, bridge, top, etc.) with three possible initial orientations shown in Fig. 1. Upon relaxation, $\mathrm{H}_{2}$ molecule has remained parallel to the surface oriented from $\mathrm{B}$ to $\mathrm{N}$ atom above the center of hexagon for each configuration at a distance of $\sim 3.5 \AA$. The binding energy $E_{b}$ is calculated as $50 \mathrm{meV}$. The binding is weak and physisorption and does not differ significantly from the binding of $\mathrm{H}_{2}$ to SWNT. Consequently, absorbed $\mathrm{H}_{2}$ molecules can be desorbed at room temperature. Earlier, Jhi and Kwon ${ }^{34}$ calculated $E_{b}\left(\mathrm{H}_{2}\right)$ as $100 \mathrm{meV}$ for $(10,0)$ SWBNT, where $\mathrm{H}_{2}$ is placed above the center of hexagon at a distance of $3.0 \AA$. Clearly, bare SWBNTs are not suitable for hydrogen storage applications.

Next, we consider SWBNT functionalized by Ti. Ti is bound to $(8,0)$ SWBNT surface with $E_{b}$ of $1.09 \mathrm{eV}$ without facing energy barrier. This displays a lower binding energy than that is obtained for SWNT $(2.2 \mathrm{eV}),{ }^{35,36}$ but it is still strong. Among possible adsorption sites, the most energetic one is found to be near the hollow site, where $\mathrm{Ti}$ atom is slightly displaced toward $\mathrm{B}$ and $\mathrm{N}$ atoms. The distances of $\mathrm{Ti}$ atom to the nearest $\mathrm{B}$ and $\mathrm{N}$ atoms are calculated to be 2.38 and $2.23 \AA$, respectively. We note that this adsorption site of $\mathrm{Ti}$ is different from that of SWNT. The ground state of the system is ferromagnetic with a net magnetic moment of $2.0 \mu_{B}$. According to Mulliken analysis, Ti atom donates 0.78 electron to SWBNT. Single $\mathrm{H}_{2}$ molecule attaching to the optimized SWBNT+ Ti dissociates into two $\mathrm{H}$ atoms as shown in Fig. 2. The $\mathrm{H}-\mathrm{H}$ distance $\left(d_{H-H}\right)$ increases to $3.38 \AA$, which was initially $0.74 \AA$. The ground state is still magnetic with $\mu=2 \mu_{B}$. The binding energy is calculated to be $0.93 \mathrm{eV}$ per $\mathrm{H}$ atom. This is similar to the single $\mathrm{H}_{2}$ adsorption to Ti on $\mathrm{SWNT},{ }^{8}$ where $\mathrm{H}_{2}$ also dissociates into two $\mathrm{H}$ atoms with $E_{b}=0.83 \mathrm{eV}$ and $d_{H-H}=2.71 \AA$. The total energy further decreases upon absorption of additional $\mathrm{H}_{2}$ molecules. Unlike the first adsorption, subsequent hydrogen molecules do not dissociate, but their $\mathrm{H}-\mathrm{H}$ bonds elongate from 0.74 to $0.84-0.90 \AA$. These results are consistent with both SWNT+Ti in Ref. 8 and also elongated $\mathrm{H}-\mathrm{H}$ bonds observed in metal-dihydrogen complexes. ${ }^{17}$ The average binding energy of molecularly absorbed hydrogens is $0.46 \mathrm{eV}$, and the ground state is paramagnetic. Another possible configuration similar to that of SWNT+Ti, where four (a)
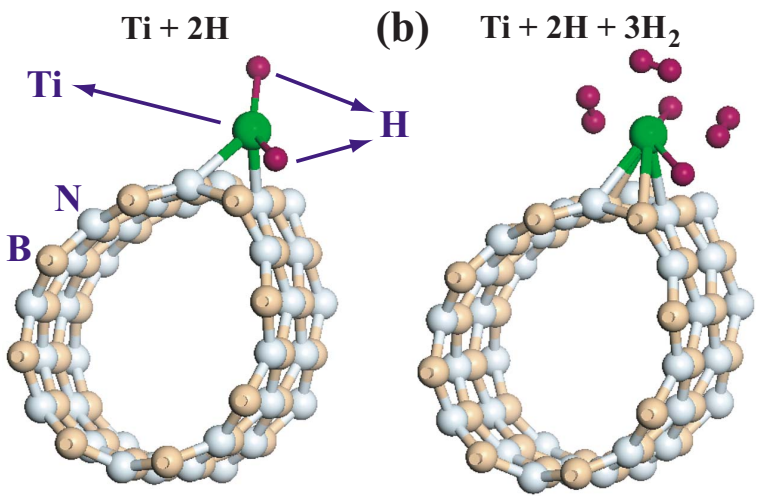

(c)

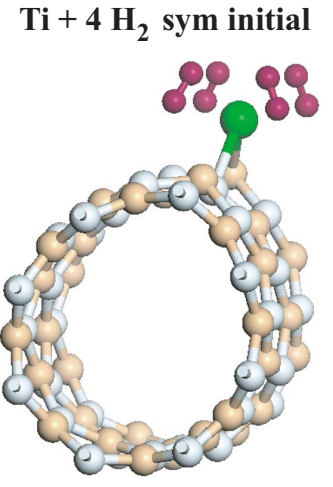

(d) $\mathrm{Ti}+2 \mathrm{H}+3 \mathrm{H}_{2}$ relaxed

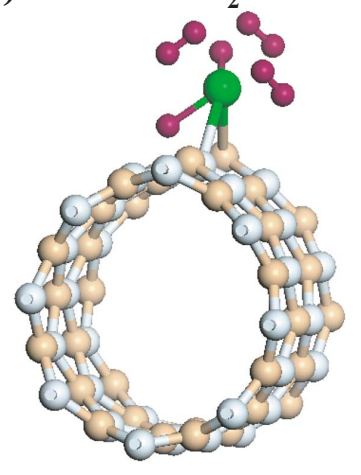

FIG. 2. (Color online) The configuration of $\mathrm{H}_{2}$ molecules absorbing to $(8,0)$ SWBNT+Ti system. (a) First $\mathrm{H}_{2}$ molecule dissociates into two $\mathrm{H}$ atom. (b) Subsequent three $\mathrm{H}_{2}$ are molecularly absorbed around Ti. (c) Possible (unrelaxed) symmetric configuration similar to that of SWNT+Ti with four $\mathrm{H}_{2}$ molecularly absorbed around Ti (Ref. 8). (d) The resulting configuration occurring upon the relaxation of $(\mathrm{c})$.

$\mathrm{H}_{2}$ molecules are molecularly absorbed as shown in Fig. 2 (c), is found to be metastable. Upon relaxation of the system, one $\mathrm{H}_{2}$ dissociated as shown in Fig. 2(d). This geometry is found to be $0.5 \mathrm{eV}$ less energetic than that is in Fig. 2(b). Our further study indicates that fifth $\mathrm{H}_{2}$ cannot be bound. In order to test the stability of the system, we first increased the distance between $\mathrm{Ti}+2 \mathrm{H}+3 \mathrm{H}_{2}$ complex and $(8,0)$ SWBNT. After relaxation, $\mathrm{Ti}+2 \mathrm{H}+3 \mathrm{H}_{2}$ complex returns to its initial configuration shown in Fig. 2(b). To further check the stability, the hydrogen atoms and molecules are also distorted from their equilibrium positions. Upon relaxation, equilibrium geometry is obtained indicating the stability of the system.

In order to increase $\mathrm{H}_{2}$ storage, we consider uniform coverage of SWBNT with Ti. First, we placed eight Ti atoms per cell uniformly at the equivalent equilibrium position obtained from the optimization of single $\mathrm{Ti}$ atom adsorbed on SWBNT, as shown in Fig. 3(a). Unfortunately, Ti atoms dimerized when they are relaxed as illustrated in Fig. 3(b). To prevent $\mathrm{Ti}$-Ti interaction leading to dimerization, we reduced the Ti coverage by placing four Ti atoms per cell as shown in Fig. 2(c). In this case, an energy barrier occurring between two Ti atoms prevented the dimerization upon relaxation. Under these circumstances, approximately $5.7 \mathrm{wt} \% \quad(3.9 \mathrm{wt} \%$ if the dissociated $\mathrm{H}$ atoms which 
(a)

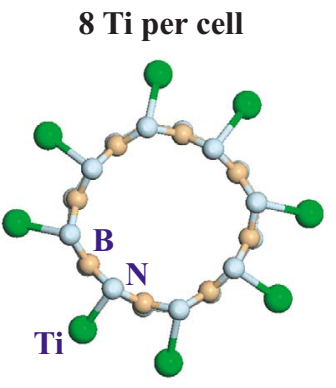

(c)

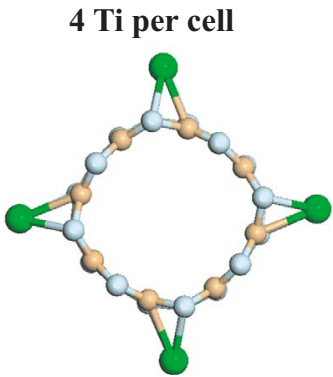

(b)

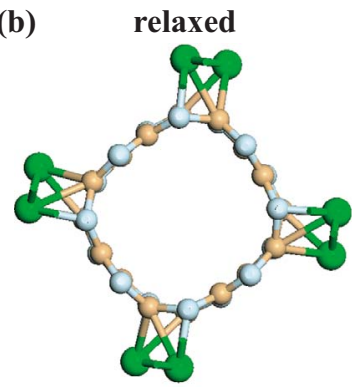

(d)

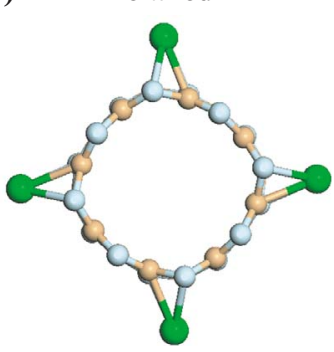

FIG. 3. (Color online) Initial and final geometries of Ti covered $(8,0)$ SWBNT. (a) Eight Ti per cell coverage (unrelaxed). (b) Eight Ti per cell coverage upon relaxation. (c) Four Ti per cell coverage (unrelaxed). (d) Four Ti per cell coverage upon relaxation.

strongly bound to $\mathrm{Ti}$ atom are not counted) can be stored. The capacity may further be increased considering internal adsorption, for $(n, 0)$ SWBNTs having relatively larger radius.

\section{II. $\mathrm{H}_{2}$ ABSORPTION ON B- AND Be-SUBSTITUTED $(8,0)$ SINGLE WALL CARBON NANOTUBE}

Earlier studies ${ }^{7}$ reveal that the interaction of $\mathrm{H}_{2}$ with the outer surface of SWNT is very weak. The binding energy is calculated to be $\sim 30 \mathrm{meV}$ without van der Walls (vdW) interaction and is estimated to be $50-60 \mathrm{meV}$ if $\mathrm{vdW}$ attraction is included. ${ }^{7}$ This interaction cannot be enhanced significantly by increasing the curvature of surface through radial deformation ${ }^{7}$ due to the fact that $\mathrm{H}_{2}-\mathrm{SWNT}$ distance is large. Other studies also showed that the physisorption of $\mathrm{H}_{2}$ cannot occur in the inner wall of SWNT. ${ }^{37}$

It is argued that $\mathrm{H}_{2}$ absorption energy is enhanced by $\mathrm{B}$ doping of fullerenes. B-doped fullerenes are known to exist experimentally. It was reported that $\mathrm{B}$ atoms could replace $\mathrm{C}$ to form $\mathrm{C}_{54} \mathrm{~B}_{6}$ and $\mathrm{C}_{48} \mathrm{~B}_{12}$ (Ref. 38) fullerenes. It is also shown that $\mathrm{C}_{59} \mathrm{~B}$ and $\mathrm{C}_{58} \mathrm{~B}_{2}$ could be synthesized even in macroscopic quantities and are stable above room temperature. ${ }^{39,40}$ Since SWNTs have similarities to fullerenes, it is interesting to consider B- or Be-substituted SWNTs.

As a first step [Fig. 4(a)], the substitution energy of B (or $\mathrm{Be})$ in $(8,0)$ SWNT $\left(\mathrm{C}_{32}\right)$ is calculated according to the expression $E_{\text {sub }}=E_{T}\left[\operatorname{SWNT}\left(\mathrm{C}_{32}\right)\right]+E_{T}[X]-E_{T}\left[\operatorname{SWNT}\left(\mathrm{C}_{31}\right)\right.$ $+X]-E_{T}[\mathrm{C}]$, where $E_{T}\left[\operatorname{SWNT}\left(\mathrm{C}_{31}\right)+X\right]$ is the total energy of the system where one of $\mathrm{C}$ atoms is replaced by $X(X=\mathrm{B}$ or $\mathrm{Be}), E_{T}\left[\mathrm{SWNT}\left(\mathrm{C}_{32}\right)\right]$ is the total energy of bare $(8,0) \mathrm{SWNT}$, $E_{T}[X]$ and $E_{T}[\mathrm{C}]$ are the free atom energies of $X$ and carbon

(a)

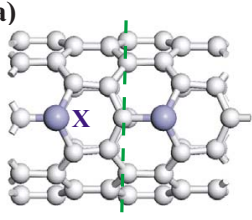

(b)

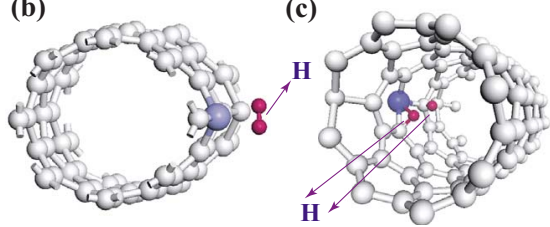

FIG. 4. (Color online) The structures of $\mathrm{H}_{2}$ absorption on $\mathrm{Be}$ (or B) substituted $(8,0)$ SWNT. (a) The final geometry of $\operatorname{SWNT}\left(\mathrm{C}_{31} X\right)$ system where one of the carbon atoms of $(8,0)$ SWNT is replaced by $X(X=\mathrm{B}$ or $\mathrm{Be})$. (b) External and (c) internal absorptions of $\mathrm{H}_{2}$ molecule on $\mathrm{SWNT}\left(\mathrm{C}_{31} X\right)$ system. $\mathrm{H}_{2}$ is molecularly absorbed for external case but dissociates into two atoms and binds to carbons for internal adsorption.

atom, respectively. Accordingly, $E_{s u b}(\mathrm{~B})$ is found to be $-3.27 \mathrm{eV} . E_{\text {sub }}<0$ indicates endothermic process and substitution requires energy. Next, we placed $\mathrm{H}_{2}$ on top of substituted $\mathrm{B}$ atom at different initial sites as shown in Fig. 4(b) and fully relax the system. $E_{b}$ is calculated as $25 \mathrm{meV}$, which is also very weak. Thus, we conclude that $\mathrm{B}$ substitution does not enhance $\mathrm{H}_{2}-\mathrm{SWNT}$ interaction. It is noted that Zhao et al. ${ }^{9}$ calculated $E_{b}=-30 \mathrm{meV}$ for B substituted fullerene $\left(\mathrm{C}_{36}\right)$ but obtained bonding state with $E_{b}=390 \mathrm{meV}$ using localdensity approximation (LDA). Our LDA calculations result to $E_{b}=61 \mathrm{meV}$ for the SWNT case.

Next, we analyze the substitution of Be. $E_{\text {sub }}(\mathrm{Be})$ is calculated to be $-9.36 \mathrm{eV}$, which is quite high indicating the adversity of Be substitution. Note, however, that due to concerted process, the energy required to substitute both B and Be into SWNT can be smaller than these substitution energies. For this case, $\mathrm{H}_{2}$ absorption on top of Be [Fig. 4(b)] yields $0.31 \mathrm{eV}$ binding energy which is significant. The $\mathrm{Be}-\mathrm{H}$ distance is calculated to be $2.0 \AA$, and $\mathrm{H}-\mathrm{H}$ bond length remained $0.77 \AA$. The slight elongation of $\mathrm{H}-\mathrm{H}$ bond also indicates that the character of the bonding is physisorption. Attempts to absorb additional $\mathrm{H}_{2}$ molecules have failed. In the case of internal adsorption, $\mathrm{H}_{2}$ molecule is dissociated and $\mathrm{H}$ atoms are bound to $\mathrm{C}$ atoms in the vicinity of $\mathrm{Be}$ as illustrated in Fig. 4(c). Similar to the fullerene case, several Be atoms may be substituted into the $(8,0)$ SWNT. We found that four Be atoms can be substituted without damaging the tubular structure $\left(\mathrm{C}_{28} \mathrm{Be}_{4}\right)$. However, when the number of $\mathrm{Be}$ atom increases, the tubular form is destroyed. Each of the four substituted $\mathrm{Be}$ atom can bind one $\mathrm{H}_{2}$ molecule. The average binding energy of $\mathrm{H}_{2}$ molecules is calculated as $0.40 \mathrm{eV}$. The Mulliken analysis indicates that Be donates 1.2 electrons to the nearest three $\mathrm{C}$ atoms $(\sim 0.4$ electron to each). The hydrogen storage capacity of $\mathrm{C}_{28} \mathrm{Be}_{4} \mathrm{H}_{8}$ system is calculated to be $\sim 2.4 \mathrm{wt} \%$. The substitution of Be into fullerene $\left(\mathrm{C}_{36}\right)$ also significantly enhanced the absorption energy of $\mathrm{H}_{2}$ molecules, and the binding energy was calculated to be $0.37 \mathrm{eV}$ with GGA and 0.65 with LDA. ${ }^{9}$ Note that the present binding energies of $\mathrm{H}_{2}$ to $\mathrm{B}$ - or Be-substituted SWNTs calculated by GGA are consistently $\sim 0.35 \mathrm{eV}$ lower than those calculated for $\mathrm{C}_{36}$ fullerenes using LDA. ${ }^{9}$

\section{CONCLUSION}

In conclusion, using first-principles DFT calculations, we found that the interaction of $\mathrm{H}_{2}$ with SWBNT is very weak. 
However, SWBNT can be functionalized by $\mathrm{Ti}$ atom and single Ti can bind up to four $\mathrm{H}_{2}$ molecules, one is dissociated and the remaining three are molecularly absorbed. Accordingly, the hydrogen storage capacity of the system ranges between 3.9 and $5.7 \mathrm{wt} \%$. Our calculations also reveal interaction of $\mathrm{H}_{2}$ with B- and Be-doped SWNTs. The substitution of both atoms is endothermic. Once Be is substituted, it significantly enhances $\mathrm{H}_{2}$ binding. Four $\mathrm{Be}$ atoms can be substituted without destroying the tubular structure which reaches $2.4 \mathrm{wt} \%$ hydrogen storage capacity.

\section{ACKNOWLEDGMENT}

This work was supported by TÜBİTAK under Grant No. TBAG-104T536. *ciraci@fen.bilkent.edu.tr

${ }^{1}$ R. Coontz and B. Hanson, Science 305, 957 (2004).

${ }^{2}$ G. W. Crabtree, M. S. Dresselhaus, and M. V. Buchanan, Phys. Today 57(12), 39 (2004).

${ }^{3}$ A. C. Dillon, K. M. Jones, T. A. Bekkendahl, C. H. Kiang, D. S. Bethune, and M. J. Heben, Nature (London) 386, 377 (1997).

${ }^{4}$ S. P. Chan, G. Chen, X. G. Gong, and Z. F. Liu, Phys. Rev. Lett. 87, 205502 (2001).

${ }^{5}$ O. Gulseren, T. Yildirim, and S. Ciraci, Phys. Rev. Lett. 87, 116802 (2001).

${ }^{6}$ W.-Q. Deng, X. Xu, and William A. Goddard III, Phys. Rev. Lett. 92, 166103 (2004).

${ }^{7}$ S. Dag, Y. Ozturk, S. Ciraci, and T. Yildirim, Phys. Rev. B 72, 155404 (2005).

${ }^{8}$ T. Yildirim and S. Ciraci, Phys. Rev. Lett. 94, 175501 (2005).

${ }^{9}$ Y. Zhao, Yong-Hyun Kim, A. C. Dillon, M. J. Heben, and S. B. Zhang, Phys. Rev. Lett. 94, 155504 (2005).

${ }^{10}$ T. Yildirim, J. Iniguez, and S. Ciraci, Phys. Rev. B 72, 153403 (2005).

${ }^{11}$ B. Bogdanovic, M. Felderhoff, S. Kaskel, A. Pommerin, K. Schlichte, and F. Schuth, Adv. Mater. (Weinheim, Ger.) 15, 1012 (2003).

${ }^{12}$ T. Yildirim and M. R. Hartman, Phys. Rev. Lett. 95, 215504 (2005).

${ }^{13}$ Y. Zhao, A. C. Dillon, Y-H. Kim, M. J. Heben, and S. B. Zhang, Chem. Phys. Lett. 425, 273 (2006).

${ }^{14}$ N. Akman, E. Durgun, T. Yildirim, and S. Ciraci, J. Phys.: Condens. Matter 18, 9509 (2006)

${ }^{15}$ H. Lee, W. I. Choi, and J. Ihm, Phys. Rev. Lett. 97, 056104 (2006).

${ }^{16}$ E. Durgun, S. Ciraci, W. Zhou, and T. Yildirim, Phys. Rev. Lett. 97, 226102 (2006).

${ }^{17}$ G. J. Kubas, Metal Dihydrogen and Bond Complexes Structure, Theory, and Reactivity (Kluwer Academic, Dordrecht/Plenum, New York, 2001).

${ }^{18}$ Y.-H. Kim, Y. Zhao, A. Williamson, M. J. Heben, and S. B. Zhang, Phys. Rev. Lett. 96, 016102 (2005).

${ }^{19}$ C. Tang, Y. Bando, X. Ding, S. Qi, and D. Goldberg, J. Am. Chem. Soc. 124, 14550 (2002).

${ }^{20}$ R. Ma, Y. Bando, H. Zhu, T. Sato, C. Xu, and D. Wu, J. Am. Chem. Soc. 124, 7672 (2002).

${ }^{21}$ S. S. Han, S. H. Lee, J. K. Kang, and H. M. Lee, Phys. Rev. B 72,
113402 (2005).

${ }^{22}$ N. Koi and T. Oku, Solid State Commun. 131, 121 (2004).

${ }^{23}$ Numerical computations have been carried out by using VASP software: G. Kresse and J. Hafner, Phys. Rev. B 47, R558 (1993); G. Kresse and J. Furthmuller, ibid. 54, 11169 (1996).

${ }^{24}$ P. Hohenberg and W. Kohn, Phys. Rev. 136, B864 (1964); W. Kohn and L. J. Sham, ibid. 140, A1133 (1965).

${ }^{25}$ D. Vanderbilt, Phys. Rev. B 41, R7892 (1990).

${ }^{26}$ J. P. Perdew, J. A. Chevary, S. H. Vosko, K. A. Jackson, M. R. Pederson, D. J. Singh, and C. Fiolhais, Phys. Rev. B 46, 6671 (1992).

${ }^{27}$ H. J. Monkhorst and J. D. Pack, Phys. Rev. B 13, 5188 (1976).

${ }^{28}$ M. S. Dresselhaus, G. Dresselhaus, and P. C. Eklund, Science of Fullerenes and Carbon Nanotubes (Academic, San Diego, 1966).

${ }^{29}$ E. Bengu and L. D. Marks, Phys. Rev. Lett. 86, 2385 (2001).

${ }^{30}$ X. Blase, J.-C. Charlier, A. De Vita, and R. Car, Appl. Phys. Lett. 70, 197 (1997).

${ }^{31}$ A. Loiseau, F. Willaime, N. Demoncy, G. Hug, and H. Pascard, Phys. Rev. Lett. 76, 4737 (1996).

${ }^{32}$ M. Terauchi, M. Tanaka, K. Suzuki, A. Ogino, and K. Kimura, Chem. Phys. Lett. 324, 359 (2001).

${ }^{33}$ H. J. Xiang, J. Yang, J. G. Hou, and Q. Zhu, Phys. Rev. B 68, 035427 (2003).

${ }^{34}$ S.-H. Jhi and Y.-K. Kwon, Phys. Rev. B 69, 245407 (2004). Their binding energy is $\sim 50 \mathrm{meV}$ higher than the present one due to different methods and basis sets (atomic orbitals with double zeta polarizaton) they used in calculations.

${ }^{35}$ E. Durgun, S. Dag, V. M. K. Bagci, O. Gülseren, T. Yildirim, and S. Ciraci, Phys. Rev. B 67, 201401(R) (2003).

${ }^{36}$ E. Durgun, S. Dag, S. Ciraci, and O. Gülseren, J. Phys. Chem. B 108, 575 (2004)

${ }^{37}$ S. M. Lee and Y. H. Lee, Appl. Phys. Lett. 76, 2877 (2000); Y. Ma, Y. Xia, M. Zhao, and M. Ying, Phys. Rev. B 65, 155430 (2002).

${ }^{38}$ T. Guo, C. Jin, and R. E. Smalley, J. Phys. Chem. 95, 4948 (1991).

${ }^{39}$ H.-J. Muhr, R. Nesper, B. Schnyder, and R. Kotz, Chem. Phys. Lett. 249, 399 (1996).

${ }^{40}$ B. Cao, X. Zhou, Z. Shi, Z. Gu, H. Xiao, and J. Wang, Fullerene Sci. Technol. 6, 639 (1998). 